\title{
Diacronie
}

Studi di Storia Contemporanea

$N^{\circ} 19,3 \mid 2014$

Miscellaneo

\section{Stefano Trinchese (a cura di), Turchia d'Europa, Le ragioni di un ritorno}

\section{Luca Zuccolo}

\section{Q OpenEdition}

\section{Journals}

\section{Edizione digitale}

URL: http://journals.openedition.org/diacronie/1654

DOI: 10.4000/diacronie.1654

ISSN: 2038-0925

\section{Editore}

Association culturelle Diacronie

\section{Notizia bibliografica digitale}

Luca Zuccolo, «Stefano Trinchese (a cura di), Turchia d'Europa, Le ragioni di un ritorno », Diacronie [Online], $N^{\circ}$ 19, 3 | 2014, documento 10, Messo online il 01 septembre 2014, consultato il 24 septembre 2020. URL : http://journals.openedition.org/diacronie/1654 ; DOI : https://doi.org/10.4000/ diacronie. 1654 


\section{RECENSIONE:}

\section{Stefano TRINCHESE (a cura di), Turchia d'Europa, Le ragioni di un ritorno, Messina, Mesogea, 2012, 187 pp.}

a cura di Luca ZUCCOLO *

La visita del Presidente della Repubblica di Turchia Abdüllah Gül a Roma, dal 28 al 31 gennaio 2014, ha nuovamente riaperto il dibattito sulla Turchia, sul suo ruolo nel Mediterraneo e sul suo ingresso in Europa. Tutti temi poco noti al grande pubblico italiano che si giovano accanto al recentissimo rilancio politico anche di una nuova pubblicazione quale quella curata da Stefano Trinchese per la casa editrice Mesogea.

Questa pubblicazione che si giova del contributo di ricercatori e giornalisti esperti di questioni mediterranee, turche e medio orientali, si propone, infatti, come un utile strumento per approfondire la conoscenza di un Paese, di uno Stato e di un popolo ancora sconosciuto [sic!] al pubblico italiano ed europeo, offrendo una visione chiara di quella che la Turchia è oggi e quella che potrebbe essere domani. Lo studio di questo Paese e delle sue relazioni internazionali in primis con l'Europa, è stato sicuramente ampliato e affrontato da più parti negli ultimi anni, vista l'intensificazione del peso economico della Turchia nel mercato globale e la riattivazione delle procedure di adesione all'Unione Europea. Nonostante ciò, tuttavia, pochi sono stati gli studi che negli ultimi tempi sono stati in grado di spiegare lucidamente e in modo approfondito le dinamiche interne di un paese complesso e molto articolato sia dal punto di vista politico, sia dal punto di vista sociale, come le ultime manifestazioni di piazza e il movimento Gezi Parki hanno dimostrato. Per queste ragioni i saggi presenti nel volume curato da Trinchese si pongono in primissima linea nel panorama editoriale italiano ed europeo per ciò che riguarda la Turchia e il suo presente.

L'idea e parte dei contributi vedono la luce nell'ambito del progetto PRIN 2008 «Islam e secolarizzazione nell'età contemporanea: dinamiche culturali e religiose tra 
innovazione e tradizione nel Mediterraneo orientale», condotto presso l'Università di Chieti-Pescara e si completano nel 2012 con la pubblicazione del volume Turchia d'Europa, le ragioni di un ritorno, che, fin dal titolo, mette in luce i temi di discussione ed evidenzia l'interessante approccio degli autori.

I diversi saggi, infatti, non sono schierati ne pro ne contro la Turchia, ma attraverso una limpida e oggettiva analisi dei fatti e dell'evoluzione storica del Paese propongono un equilibrato spaccato del presente e del futuro turchi; delle reali possibilità di entrare in Europa come dell'eventualità, per Ankara, di divenire una potenza regionale autonoma, senza tralasciare gli ostacoli e i colli di bottiglia ancora presenti sul cammino turco. In secondo luogo, ma non per questo meno importante, i saggi proposti leggono in modo nuovo e stimolante anche le numerose permanenze del passato ottomano e repubblicano che ancora condizionano la società, la politica e la vita della Turchia contemporanea. I lasciti ottomani e del periodo kemalista con tutti i loro strascichi sono, infatti, interpretati come caratteri peculiari della realtà turca che non possono essere sottovalutati in una corretta analisi della realtà del Paese, al pari delle relazioni con le popolazioni e le regioni turcofone del Caucaso e dell'Asia Centrale, delle relazioni con Arabi, Israeliani e Iraniani in Medio Oriente o di quelle intrattenute nelle aree Balcaniche ex-imperiali.

All'introduzione di Stefano Trinchese, che propone un quadro generale della questione turca in relazione all'Europa e mette in luce alcuni dei malintesi che stanno rallentando l'adesione della Turchia all'Unione Europea, seguono nove saggi suddivisibili in tre sezioni, ciascuna delle quali si concentra su di un aspetto rilevante di problematicità nel nostro approccio alla Turchia. La prima sessione, che potremmo definire storica, si sofferma maggiormente sulle permanenze dell'età ottomana e del primo periodo repubblicano-kemalista. In apertura si pone il saggio di Giorgio Del Zanna - ricercatore di Storia contemporanea presso l'Università Cattolica di Milano Le comunità cristiane in Turchia. Attraverso lo studio della presenza dei cristiani in Anatolia e di conseguenza del problema relativo alle minoranze nel Paese, l'autore pone in rilievo due temi importanti: la rilevanza culturale e simbolica delle comunità cristiane turche in relazione all'ingresso della Turchia in Europa e la necessità di costruire una nuova struttura sociale più pluralista e inclusiva in grado di recuperare almeno in parte lo spirito cosmopolita ottomano. Segue il saggio di Stefania De Nardis - dottore di ricerca in Storia contemporanea e collaboratrice dell'Università di ChietiPescara - La lingua turca dall'Impero alla Repubblica, in cui l'autrice analizzando la nascita del turco moderno, tra lasciti del tardo Impero Ottomano e riforma linguistica kemalista, mette in evidenza gli aspetti e le ricadute sociali avute sul paese, e ancor oggi 
percepibili, di questa trasformazione che ha influenzato non poco la mentalità e la sociabilità turche lungo tutto il XX secolo. A chiusura di questa prima parte e quasi ad anticipazione delle sezioni seguenti, il saggio di Marco Guidi - editorialista del «Messaggero» e docente di giornalismo presso l’Università di Bologna - Turchia di Atatürk e Impero Ottomano, insiste ancora sulle permanenze e sui lasciti del passato nell'attuale struttura socio-politica, ma, cosa ancor più importante, propone un'interpretazione stimolante del presente turco come di un terzo periodo rivoluzionario ${ }^{1}$. Per l'autore, il nuovo millennio guidato dai governi dell'AKP (Adalet ve Kalkınma Partisi), si sta già presentando come un periodo di deciso cambiamento il cui fine è e deve essere il superamento dei vecchi schemi ideologici e mentali al fine di rispondere alle nuove sfide poste alla Turchia e ai suoi cittadini dalla post-modernità.

La seconda sezione socio-politica è aperta dal saggio di Paola Pizzo - ricercatrice presso l'università di Chieti-Pescara - La Turchia fra Islam e laicità. Qui l'autrice propone un'analisi storica di uno degli snodi ad oggi più critici e decisivi della nuova Turchia: il rapporto con la religione islamica. Spiegati i fatti che hanno visto la marginalizzazione dell'Islam all'inizio della Repubblica e le motivazioni della reislamizzazione degli anni Ottanta, Paola Pizzo, sottolineando il ruolo chiave dell'Islam confraternale, analizza la nuova stagione dell'Islam politico che a partire dagli anni Novanta è cresciuto come soggetto socio-politico determinante nella vita politica turca. Come sottolinea l'autrice nelle conclusioni, infatti, è importante richiamare l'attenzione su:

\footnotetext{
l'esigenza di analizzare l'islam turco anche in relazione al più ampio contesto arabo e mediterraneo in cui la Turchia è inserita. In un'ottica europea, infatti, l'ascesa al potere per vie democratiche di un partito di ispirazione islamica ha posto seri interrogativi sulla capacità della Turchia di mantenersi sul cammino delle riforme necessarie per dar seguito alla domanda di adesione all'Unione europea. Osservato da sud, invece, il caso turco presenta non pochi tratti di originalità e di interesse, soprattutto per la sfida che l'Akp sta dimostrando di saper vincere agli occhi della comunità musulmana ${ }^{2}$.
}

L'importanza dell'islam politico nella realtà turca è messo in evidenza anche da Vincenzo Pergolizzi - giornalista ed esperto di Storia della Turchia - che nel suo La rivoluzione silenziosa di Erdoğan e dell'AKP analizza l'evoluzione storica di questo

\footnotetext{
${ }^{1}$ GUIDI, Marco, Turchia di Atatürk e Impero Ottomano, in TRINCHESE, Stefano (a cura di), Turchia d'Europa, Le ragioni di un ritorno, Messina, Mesogea, 2012, p. 49.

2 PIZZO, Paola, La Turchia fra Islam e laicità, in TRINCHESE, Stefano, (a cura di), op.cit., p. 72.
} 
fenomeno, dalle prime esperienze di Neçmettin Erbakan al governo di Recep Tayyip Erdoğan. Oltre a tracciare le linee guida storiografiche di vari movimenti e partiti politici di matrice islamica succedutesi dalla fine degli anni Settanta, l'autore propone un'accurata analisi del partito di governo turco mettendone in risalto i pregi e i difetti; sottolineando il carattere post-islamico di questo partito "trasversale" che sta rivoluzionando la Turchia e la sua immagine in Europa anche grazie a politiche postottomane:

A più di ottanta anni dalla fondazione della Repubblica laica e democratica di Atatürk, il neo-ottomanesimo di Erdoğan deve reinventare ex-novo moderne coordinate post-ottomane partendo dalla scelta di fondo di un integrazione progressiva, che confluisca nell'Europa Comune3.

L'ultimo saggio di questa sezione Libertà o controllo, ovvero della modernizzazione turca, proposto da Fabio Salomoni - lettore di lingua italiana alla Koç Universitesi di Istanbul - si concentra sull'altro aspetto chiave del presente turco: la società. La democratizzazione, le aperture al pluralismo e le riforme proposte dall'AKP negli ultimi anni, infatti stanno mutando nel profondo la società turca come si è potuto constatare recentemente dalle proteste di piazza Taksim. Un cambiamento sociale dovuto soprattutto ai nuovi approcci che la politica turca sta adottando relativamente ai nodi gordiani della sua storia repubblicana: il legame tra stato e forze armate; le questioni curda e alevita; le diseguaglianze socio-economiche, la necessità di avere una memoria condivisa soprattutto per quanto riguarda il problema armeno; infine, la necessità di una riforma costituzionale e di un nuovo patto sociale. Dinamiche di cambiamento che Salomoni analizza in dettaglio sottolineando gli sforzi che la società turca nel suo complesso sta facendo per superare i limiti di una visione unitaria poco inclusiva e poco plurale.

La terza e ultima parte del volume si occupa, invece, di politica estera e del ruolo che la Turchia svolge e può svolgere nello scacchiere euro-mediterraneo, medioorientale e caucasico-centroasiatico. Su quest'ultima regione e sulle relazioni con le popolazioni turcofone si concentra il saggio Turchia e Repubbliche Turcofone di Stefano Barocci - esperto di geopolitica ed economia delle aree del Mediterraneo, del Caspio-Caucaso, dell'Iran, della Turchia e del Golfo Persico.

\footnotetext{
3 PERGOLIZZI, Vincenzo, La rivoluzione silenziosa di Erdoğan e dell'AKP, in TRINCHESE, Stefano, (a cura di), op.cit., p. 89.
} 
Uno sguardo d'insieme sulla politica estera turca è fornito dal saggio di Sebastiano Sali - dottorando presso il King's College di Londra - Potenzialità e criticità della Turchia odierna, il quale insite sul tema della sicurezza e su come la Turchia rivesta un ruolo di Stato-pivot nei molteplici teatri geostrategici in cui è coinvolta.

Conclude la terza parte il saggio di Paolo Quercia - analista di relazioni internazionali e di sicurezza - La Turchia dalla Multivettorialità al riposizionamento geopolitico. Questo contributo sebbene si allinei ai precedenti a livello tematico approfondisce maggiormente la politica estera turca concentrandosi sui rapporti bilaterali tra Ankara e i suoi vicini-competitors nelle principali aree di frizione regionale e mondiale. Si osservano le relazioni divenute complicate con Israele e quelle con Mosca, si sottolinea il nuovo e inedito rapporto con l'Iran sciita e la complicata relazione con le molteplici anime dell'Iraq post-bellico, per concludere con le rinnovate relazioni turco-tedesche, che in più situazioni richiamano le storiche relazioni tra Impero ottomano e germanico durante la Belle époque.

Il volume e chiuso da una breve post fazione di Vincenzo Pergolizzi, Un dialogo mai interrotto fra Oriente e Occidente che così si conclude:

In cinquecento anni di Impero ottomano - ripeteva pochi anni fa un docente di Teologia islamica dell'Università di Istanbul, in realtà un sufi di Mevlana - di guerre e scontri, non abbiamo mai smesso, nemmeno per un giorno, di far commercio, di dialogare, di parlare e accordarci4.

Migliori parole non si potrebbero trovare per descrivere la Turchia, i suoi rapporti con l'Europa, il Mediterraneo, il Medio Oriente, le aree turcofone e con loro questo volume. I saggi qui proposti, sebbene in alcuni casi siano relativamente datati (20082009) e siano stati superati dalla quotidianità di una società in continuo mutamento evolutivo, offrono un quadro accurato del Paese, della sua società e delle sfide che sta affrontando e continua ad affrontare. Certamente, l'argomento Turchia non è esaurito, ma senza ombra di dubbio questo volume si pone in prima fila tra i lavori e gli studi editi su questo Paese. La freschezza dei contributi e la chiarezza nell'esposizione rendono ognuno dei saggi presentati fruibile ad un vasto pubblico, fornendo al contempo agli specialisti e ai cultori della materia notevoli e interessanti spunti di dibattito, discussione e approfondimento.

4 PERGOLIZZI, Vincenzo, Un dialogo mai interrotto fra Oriente e Occidente, in TRINCHESE, Stefano, (a cura di), op.cit., p. 172. 


\section{* L'autore}

Luca Zuccolo è dottore in Storia Contemporanea presso il SUM (Istituto Italiano di Scienze Umane) di Napoli. Già dottore magistrale in Storia d'Europa (Bologna, 2008), il suo campo di ricerca si rivolge allo sviluppo della modernità durante l'ultimo secolo dell'Impero Ottomano, al confronto/scontro tra modernità e tradizione in un contesto cosmopolita e allo sviluppo dei movimenti sociali che hanno preparato l'avvento della società turca contemporanea. È il referente di Diacronie per la storia turca e ottomana.

URL: < http://www.studistorici.com/2010/12/o1/luca-zuccolo >

\section{Per citare questo articolo:}

ZUCCOLO, Luca, «Recensione: Stefano TRINCHESE (a cura di), Turchia d'Europa, Le ragioni di un ritorno, Messina, Mesogea, 2012, 187 pp.», Diacronie. Studi di Storia Contemporanea, 29/09/2014, URL:< http://www.studistorici.com/2014/09/29/zuccolo2_numero_19/ >

Diacronie Studi di Storia Contemporanea $\beta$ www.diacronie.it

Risorsa digitale indipendente a carattere storiografico. Uscita trimestrale. redazione.diacronie@hotmail.it

Comitato di redazione: Jacopo Bassi - Luca Bufarale - Elisa Grandi - Deborah Paci - Fausto Pietrancosta - Matteo Tomasoni - Luca Zuccolo

Diritti: gli articoli di Diacronie. Studi di Storia Contemporanea sono pubblicati sotto licenza Creative Commons 2.5 Possono essere riprodotti a patto di non modificarne i contenuti e di non usarli per fini commerciali. La citazione di estratti è comunque sempre autorizzata, nei limiti previsti dalla legge. 